\title{
Investigation of High Burnup Ceramic Fuel Microstructure at Idaho National Laboratory
}

\author{
Fabiola Cappia
}

May 2020

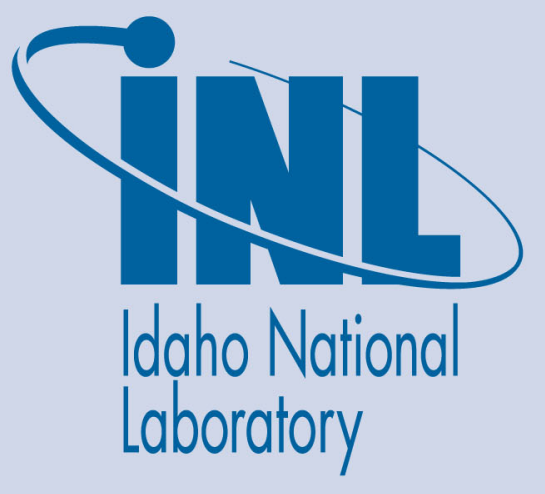

The INL is a U.S. Department of Energy National Laboratory operated by Battelle Energy Alliance 


\title{
Investigation of High Burnup Ceramic Fuel Microstructure at Idaho National Laboratory
}

\author{
Fabiola Cappia
}

May 2020

Idaho National Laboratory Idaho Falls, Idaho 83415

http://www.inl.gov

\author{
Prepared for the \\ U.S. Department of Energy \\ Office of Nuclear Energy, , Office of Nuclear Energy \\ Under DOE Idaho Operations Office \\ Contract DE-AC07-05ID14517, DE-AC07-05ID14517, DE-AC07-05ID14517
}




\section{Investigation of High Burnup Ceramic Fuel Microstructure at Idaho National Laboratory}

F. Cappia, B. Kombaiah, E. Perez ${ }^{*}$, T. Pavlov, A. Winston, F. Di Lemma, J. Madden, B. Miller, F. Teng, T. Yao, D. Murray

Characterization and Advanced Post-irradiation Examinations Division, Idaho National Laboratory

K. Mo

Argonne National Laboratory

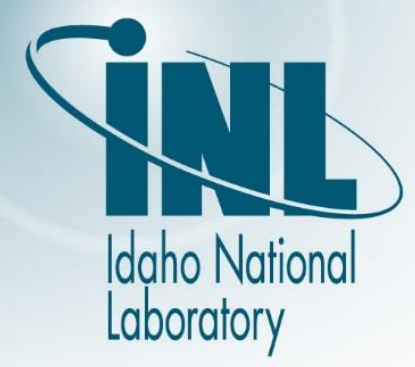

P. Lei, A. B. Mohamad, B. Gong, J. Lian

Rensselaer Polytechnic Institute

${ }^{*}$ Currently at Savannah River National Laboratory 


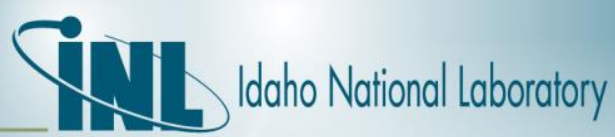

\section{Outline}

- Introduction

- The High Burnup Structure (HBS)

- Microstructural modifications due to irradiation: implications for fuel performance

- Results highlights

- High burnup commercial fuel advanced microscopy

- Separate effect testing: the role of initial microstructure in HBS formation

- Summary 


\section{Irradiation-induced changes}

- Thermo-physical properties

- Dimensional changes

- Fuel Swelling

- Axial Growth

- Cladding creep

- Fission gas production and release (pin pressure)

- Fuel restructuring (central hole formation), cracking, densification

- Constituent redistribution

- Fuel cladding chemical/mechanical interaction

- Cladding/materials corrosion

- High Burnup Structure (HBS) formation

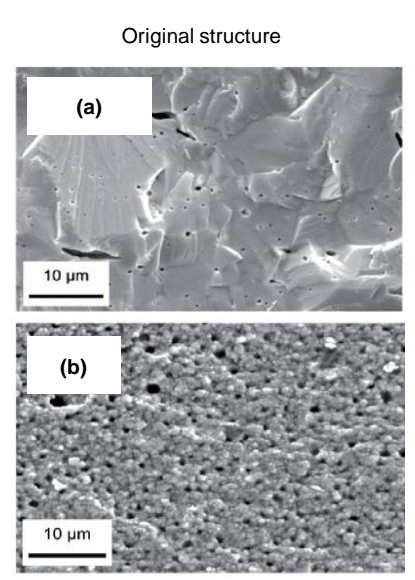

High Burnup Structure
Local enrichment in ${ }^{239} \mathrm{Pu}$ at pellet periphery takes place

$\downarrow$

1. Higher local fission density, high radiation damage

2. Limited thermal recovery

A modified structure is formed at the pellet periphery

SEM micrographs showing fuel fracture surfaces: (a) unirradiated fuel; (b) fuel with local burn-up of $\sim 75 \mathrm{GWd} / \mathrm{tHM}$

U-10Zr AFC campaign

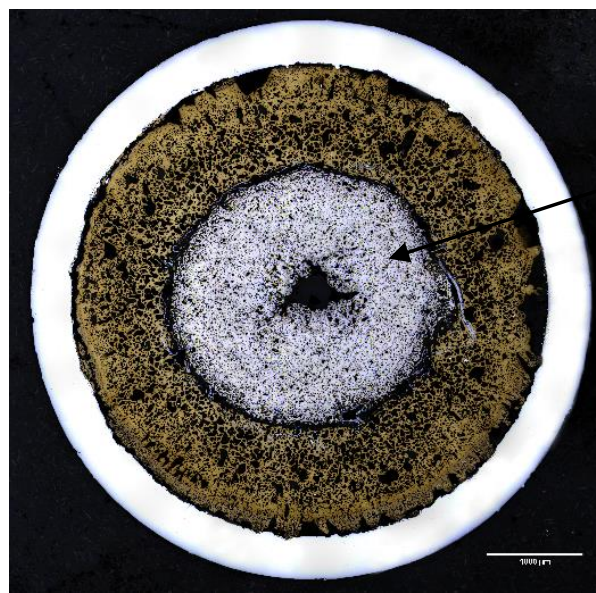

Zr-rich phase

Radial redistribution of components 


\section{Microstructural modifications: High Burnup Structure (HBS)}

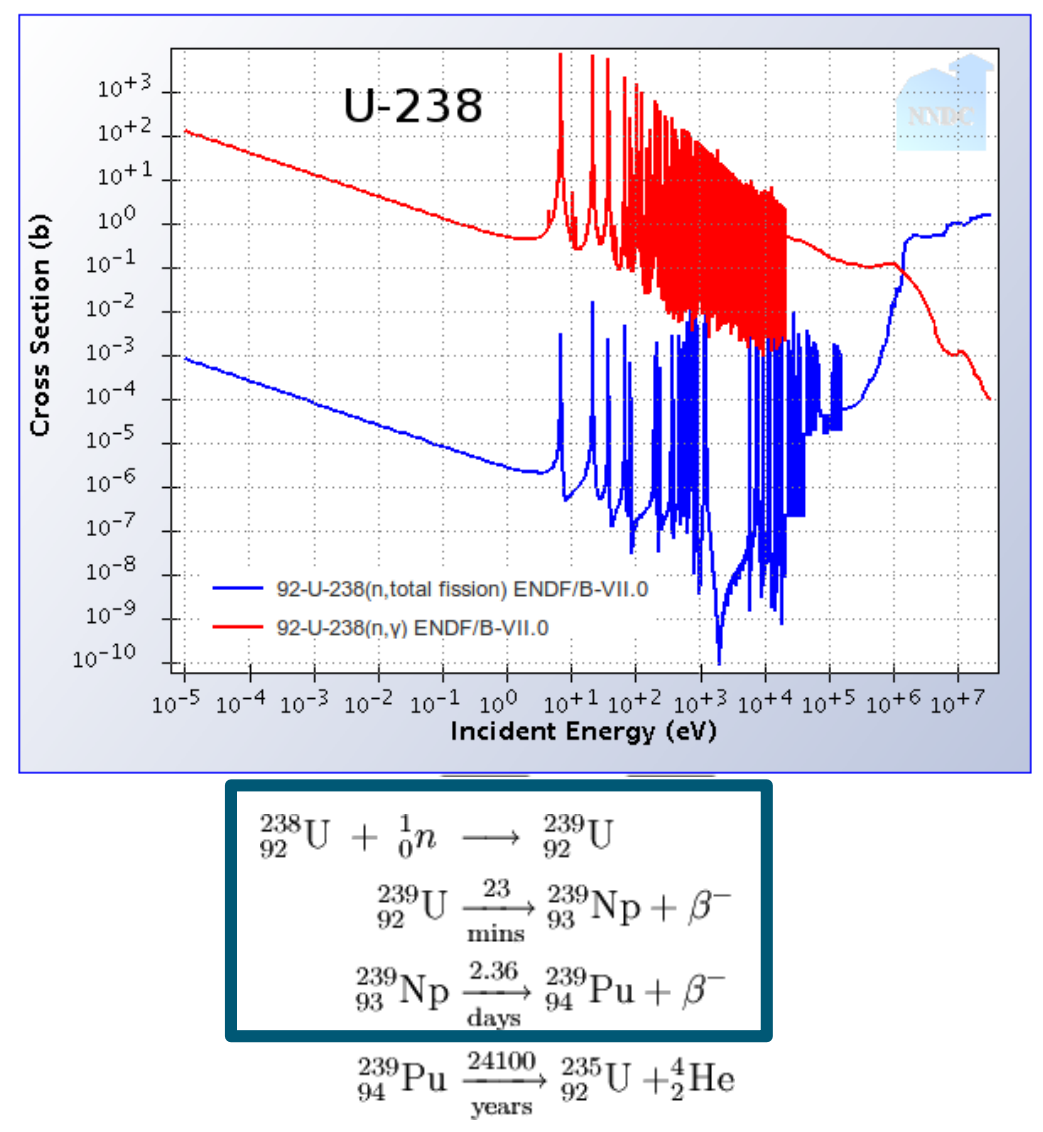

Local enrichment in ${ }^{239} \mathrm{Pu}$ at pellet periphery takes place

$\downarrow$

1. Higher local fission density, high radiation damage

$+$

2. Limited thermal recovery

$=$

A modified structure is formed at the pellet periphery

Phenomenon primarily occurring in $\mathrm{UO}_{2} \mathrm{LWR}$ fuels 


\section{HBS summary}

\section{- Characteristics}

- Grain subdivision/recrystallization

- Formation of micron-sized intergranular closed pores

- Depletion of fission gases from the fuel matrix

- Conditions for formation

- $\mathrm{T}_{\text {irradiation }} \leq 1000^{\circ} \mathrm{C}$ (approximately)

- Local burnup $\geq 50-60 \mathrm{GWd} / \mathrm{tHM}$

- Large impact on fuel properties (hence, performance)

- Mechanical $\rightarrow$ increased porosity reduces load-bearing area

- Porosity increase $\rightarrow$ swelling (stress on cladding) and decreases thermal conductivity

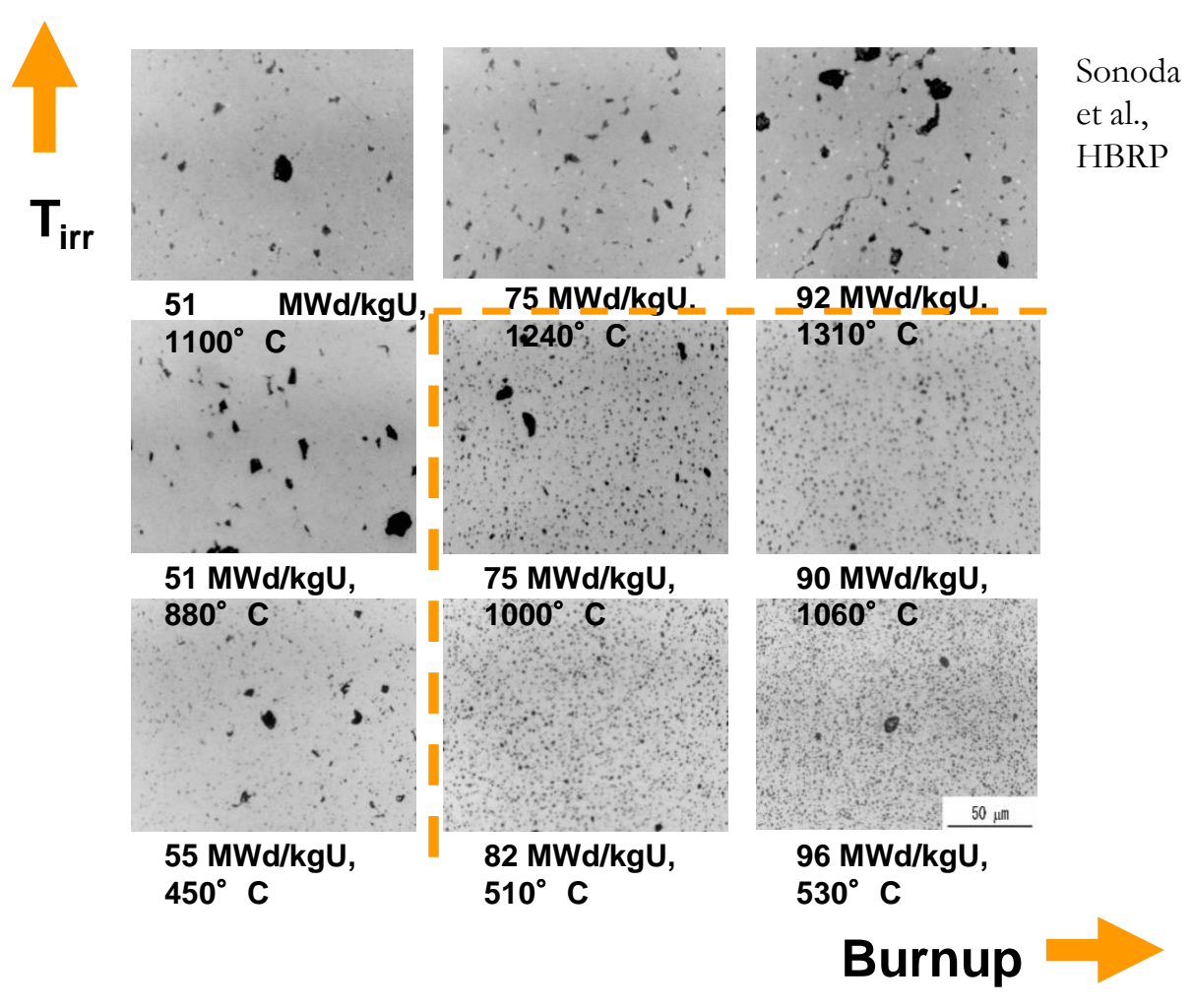




\section{Motivation for multi-scale investigation}

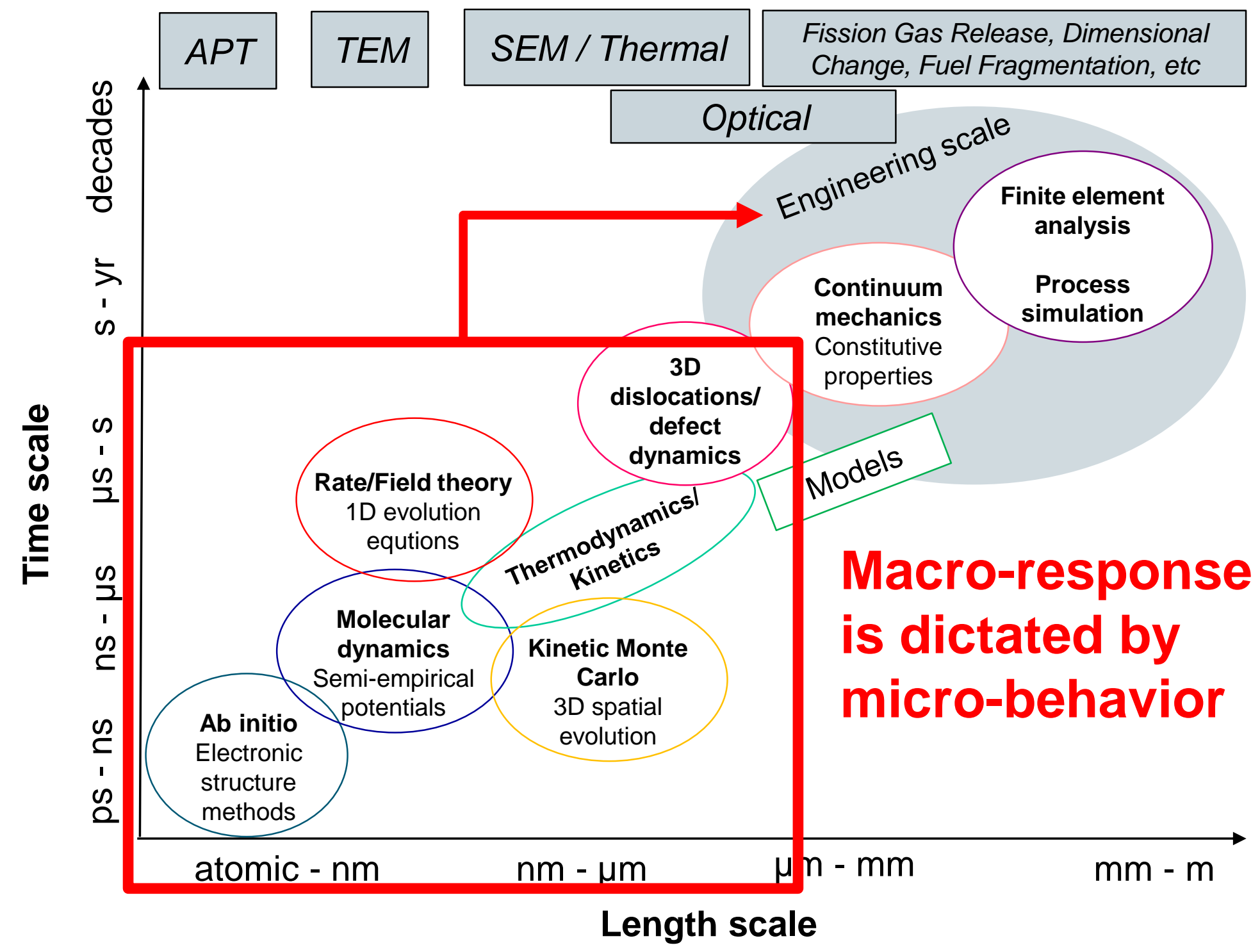




\section{Challenges in microstructural characterization of nuclear fuels}

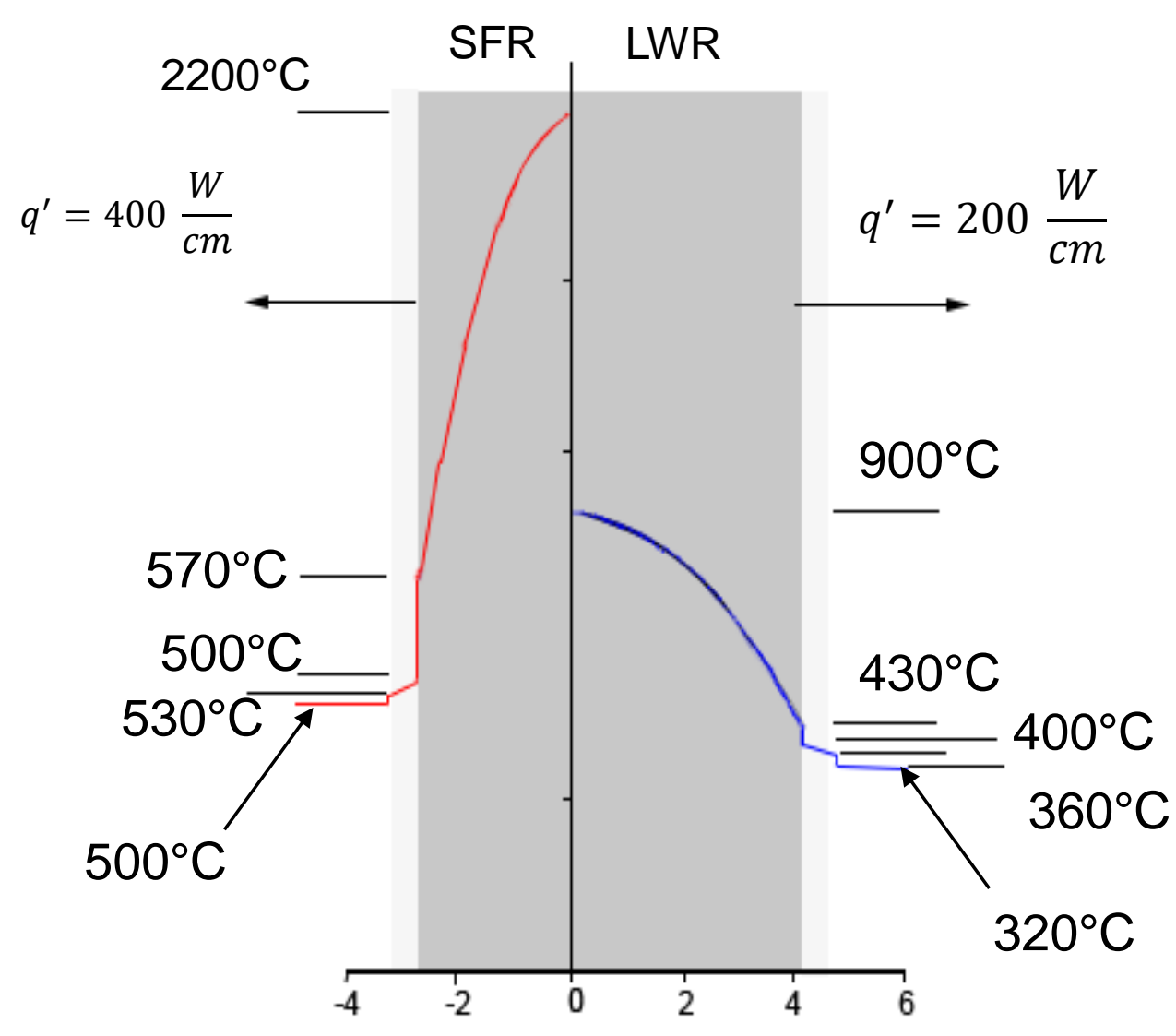

Fuel radius (mm)

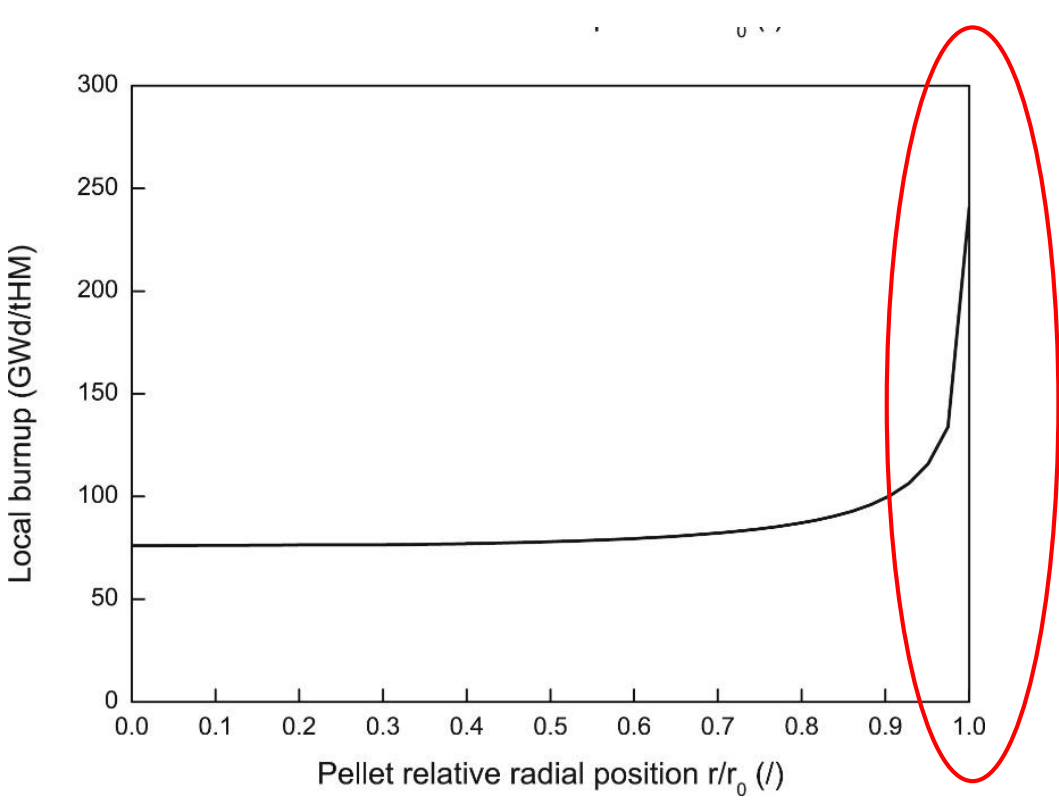

Thermal and burnup gradients require analyses with the same spatial resolution, but keeping statistical significance 


\section{Characterization
radius: porosity}

- Advanced microscopy using SEM/FIB/EBSD is the right tool to tackle the radial heterogeneities

- Experimental campaign on a high burnup (rod average $72.9 \mathrm{GWd} / \mathrm{tHM}$ ) for characterization before TREAT LOCA safety testing
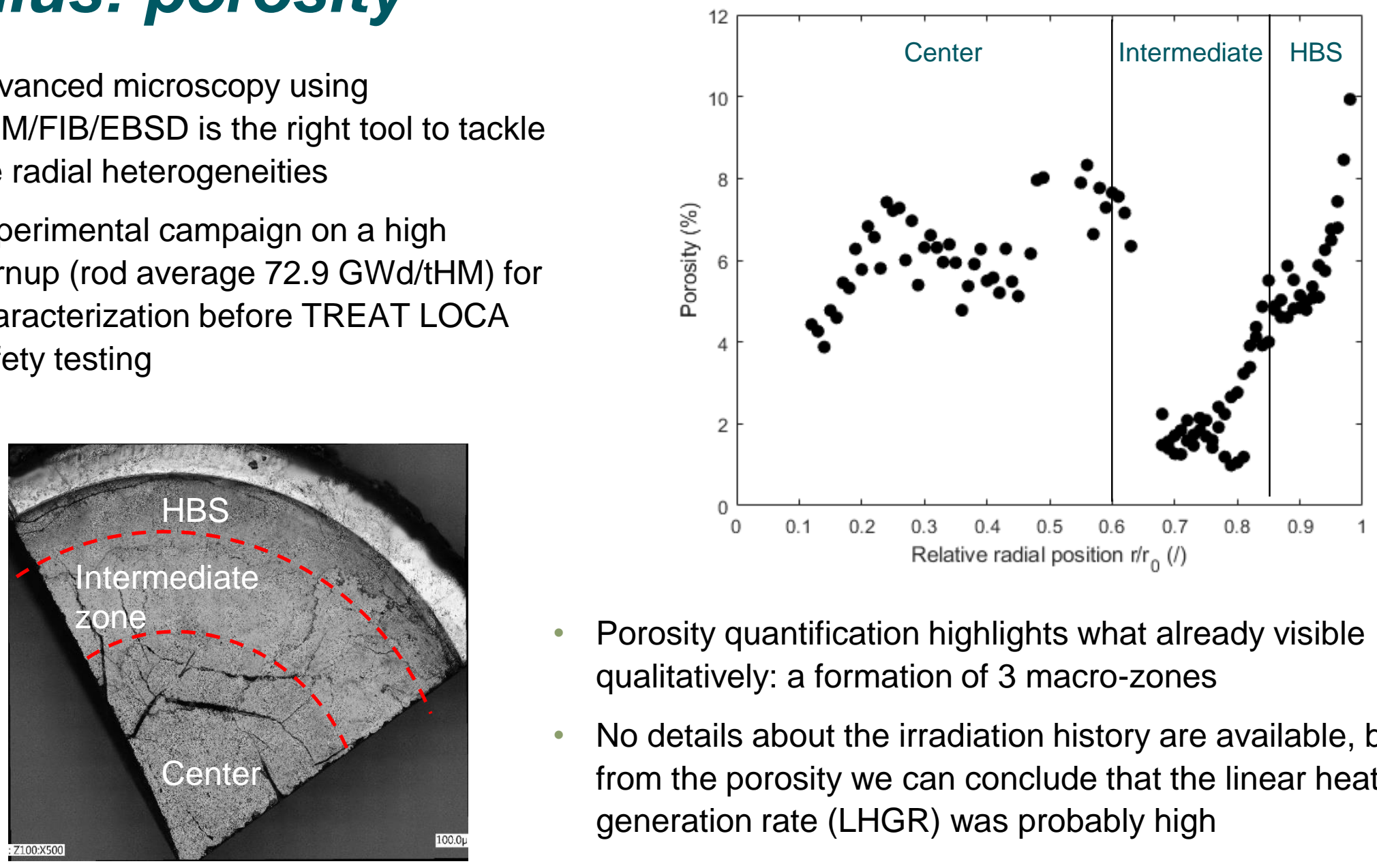

- Porosity quantification highlights what already visible qualitatively: a formation of 3 macro-zones

- No details about the irradiation history are available, but from the porosity we can conclude that the linear heat generation rate (LHGR) was probably high 


\section{Characterization of microstructure along the radius: SEM and EBSD (1/2)}
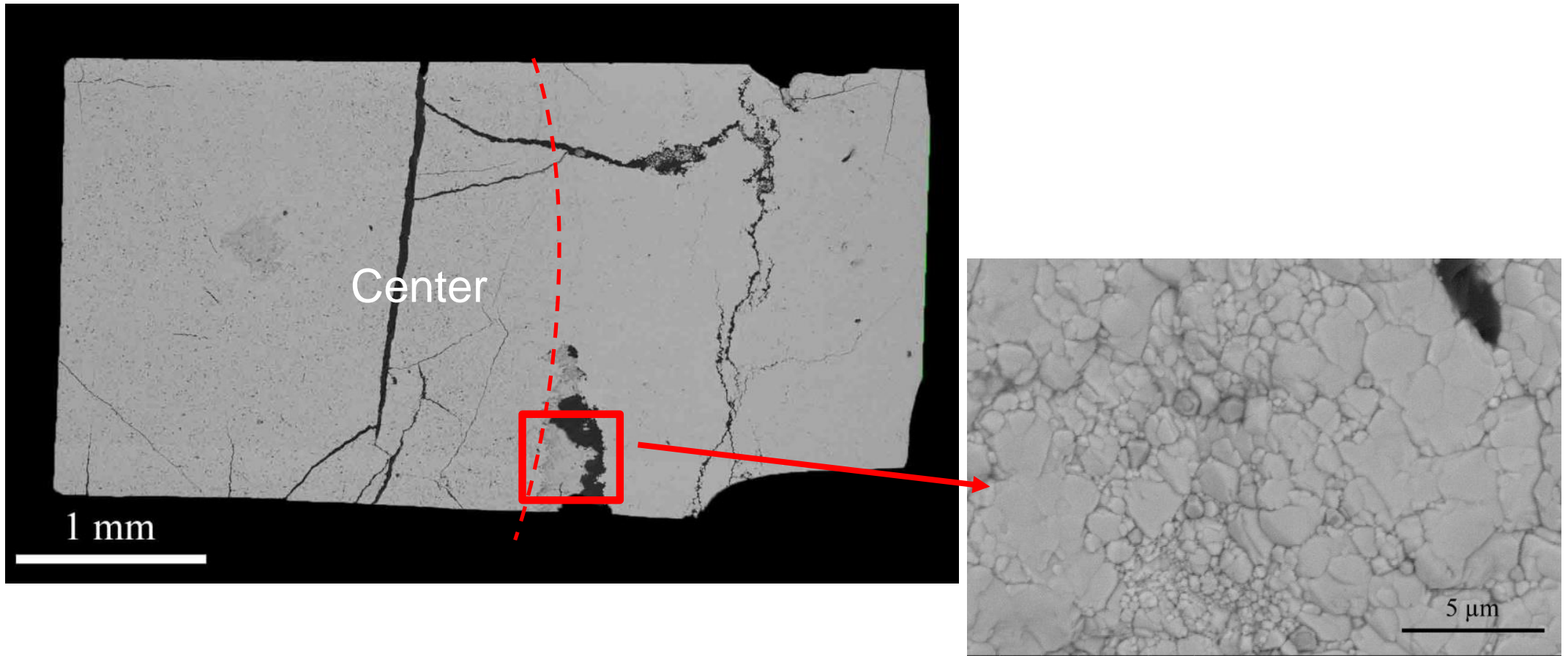

- Restructuring at the pellet periphery is a very well-known phenomenon

- But also intermediate regions start to develop grain restructuring 


\section{Characterization of microstructure along the radius: SEM and EBSD (2/2)}
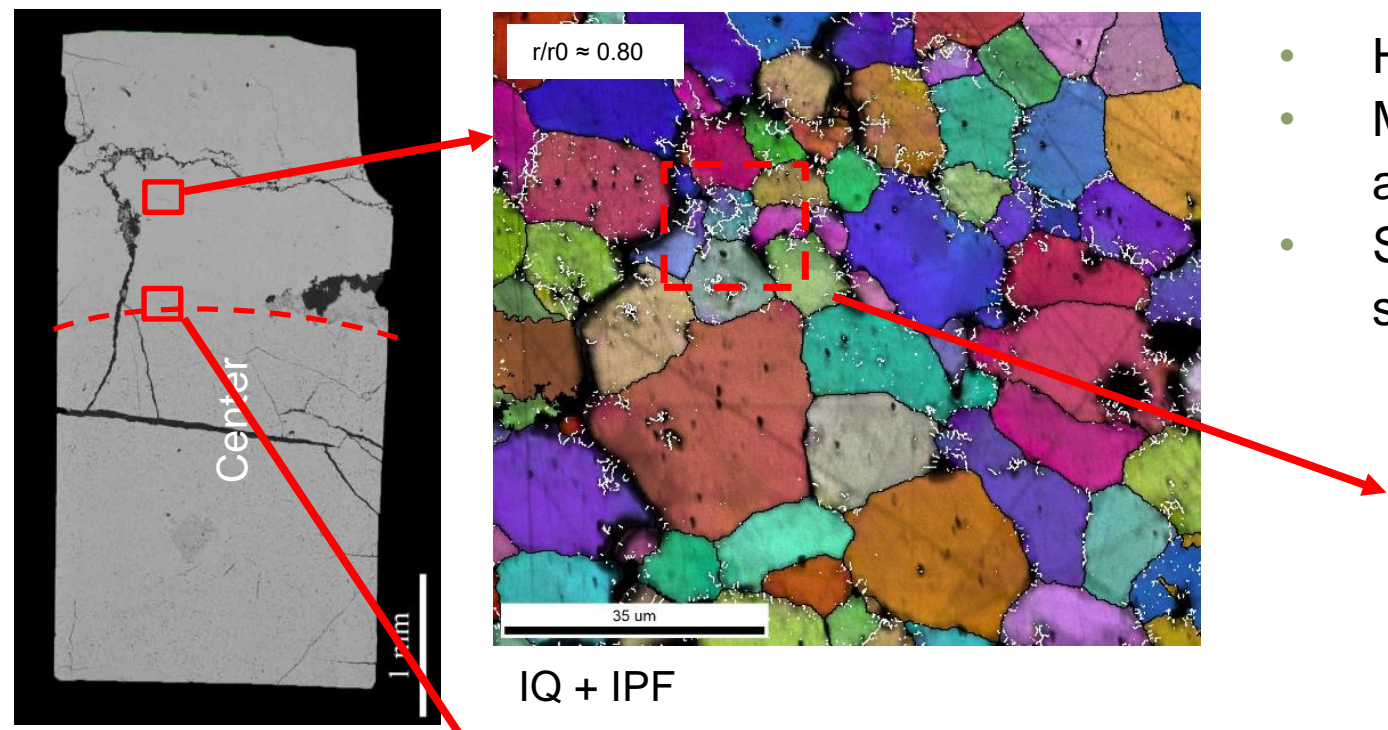

$I Q+I P F$
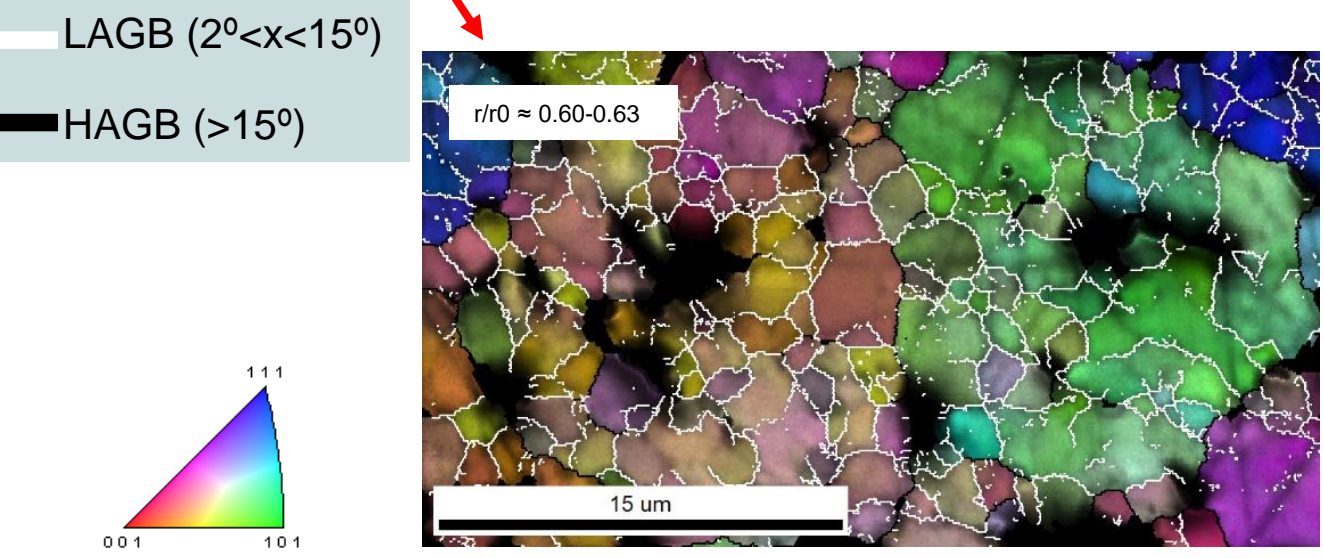

$I Q+K A M$ map $<15^{\circ}$

HAGB dominated

Most LAGB are noise from porosity and around cracking

Some degree of subdivision might occur starting on grain boundaries (very limited)

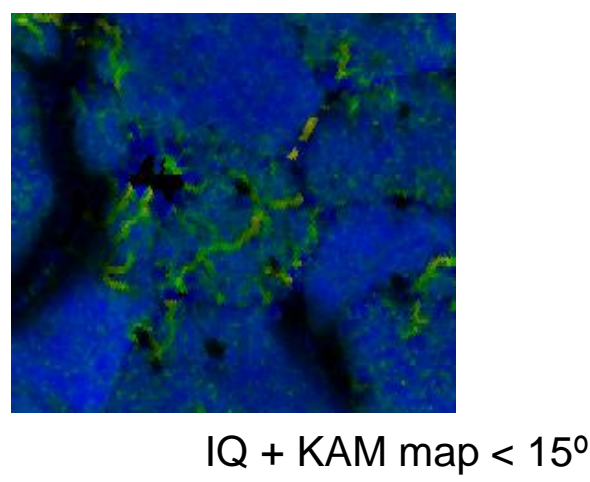

$I Q+I P F$

LAGB dominated HAGB are associated with original grain boundaries 


\section{Comparison with another high burnup sample}

$72.9 \mathrm{GWd} / \mathrm{tHM}$
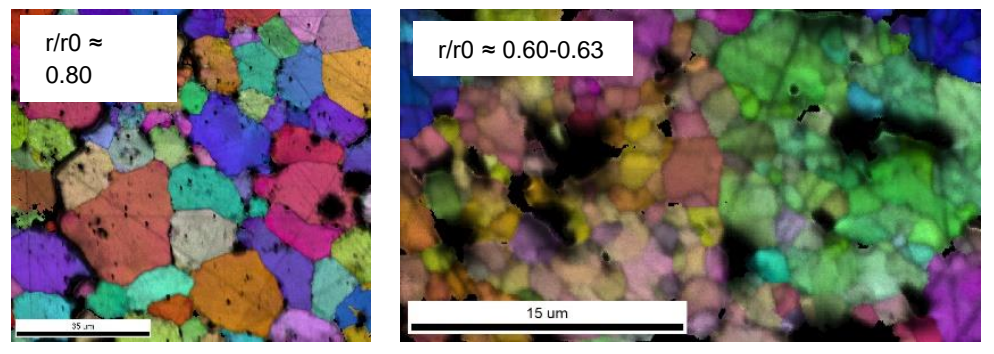

$72 \mathrm{GWd} / \mathrm{tHM}$

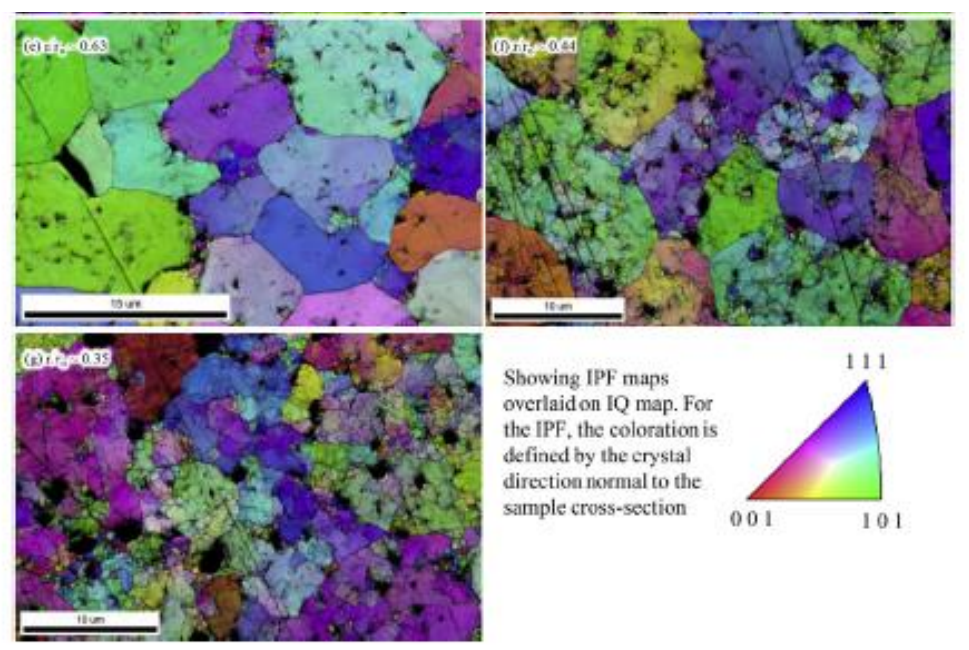

Gerczak et al., JNM 509 (2018), p. 245-259
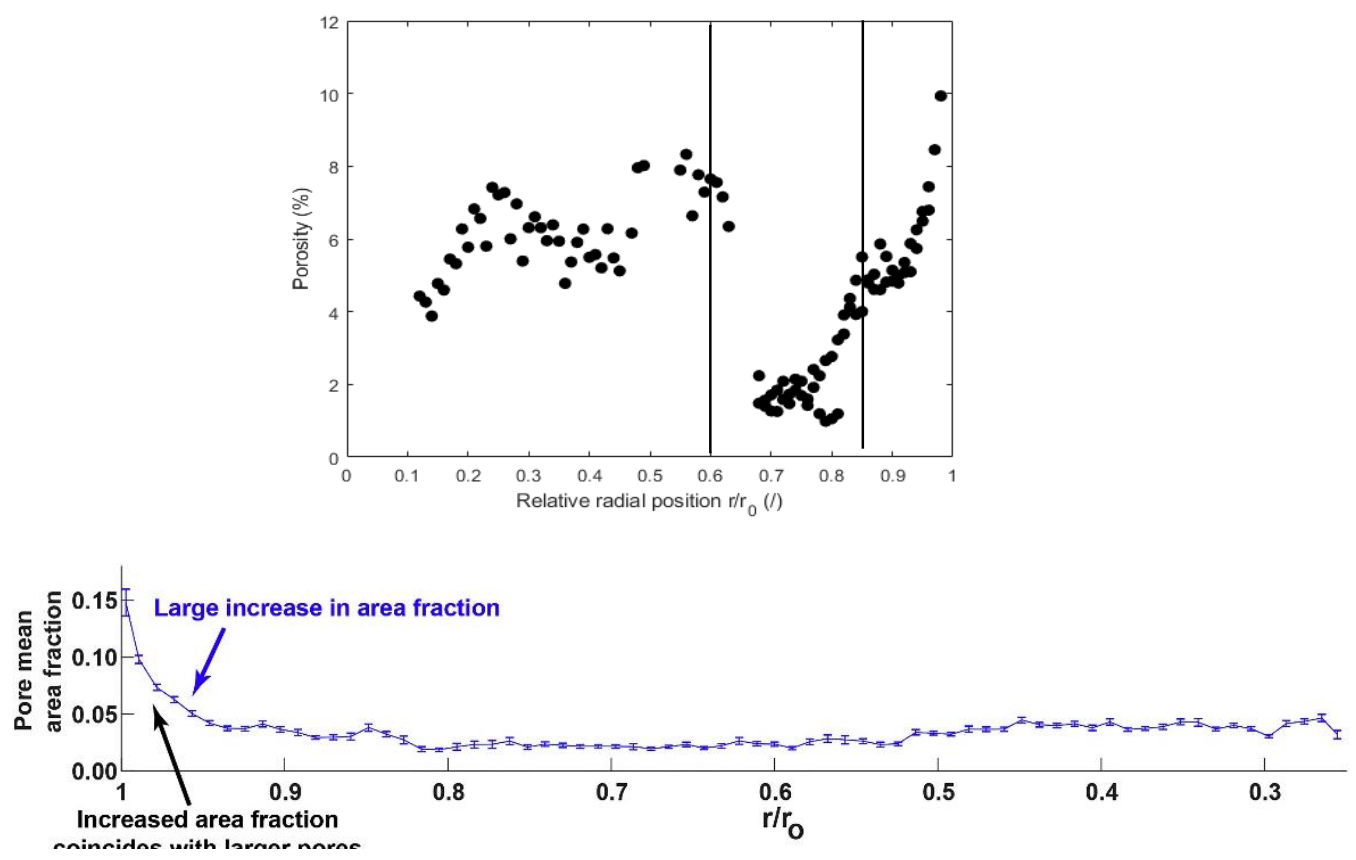

- Same burnup, but different histories lead to totally different grain structure

- Too many variables are involved to provide a clear understanding of the observations - separate effect testing needed 


\section{High energy Xe ion-irradiation to understand HBS formation}

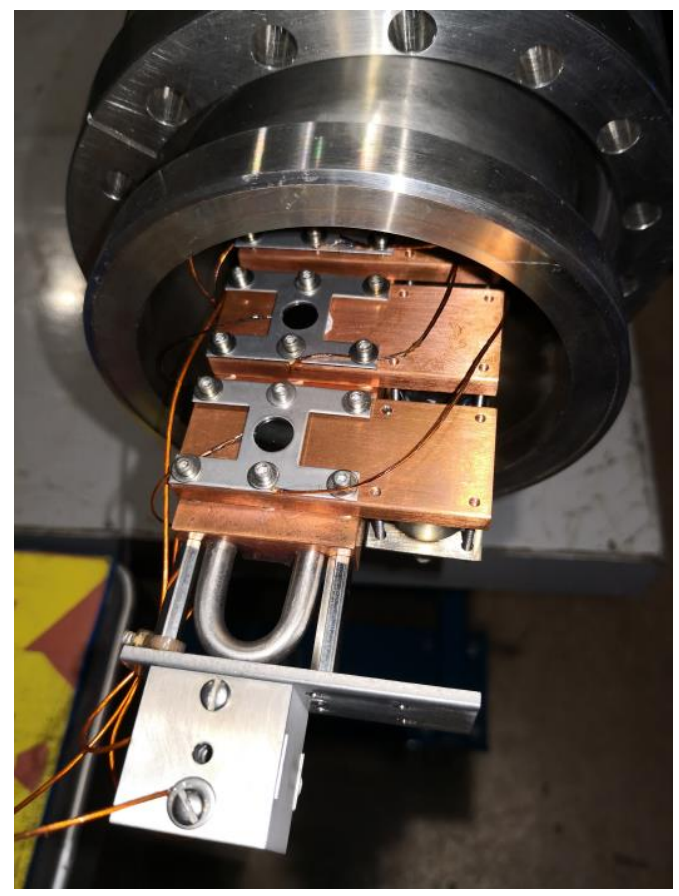

\begin{tabular}{|l|c|c|}
\hline & Sample A & Sample B \\
\hline Initial grain size $(\mu \mathrm{m})$ & 10 & 70 \\
\hline $\begin{array}{l}\text { Irradiation } \\
\text { temperature }\left({ }^{\circ} \mathrm{C}\right)\end{array}$ & 350 & 500 \\
\hline $\begin{array}{l}\text { Ion fluence }\left(\mathrm{ion} / \mathrm{cm}^{2}\right) \\
\text { dpa (SRIM } \\
\text { calculations) }\end{array}$ & $7.22 \times 10^{17}$ & $7.94 \times 10^{17}$ \\
\hline$\%$ FIMA & 1357 & 1492.7 \\
\hline
\end{tabular}

Samples fabricated at RPI using SPS

Samples irradiated at ANL ATLAS beamline with 84

$\mathrm{MeV}$ Xe 


\section{High energy Xe ion-irradiation to understand HBS formation (1/4)}

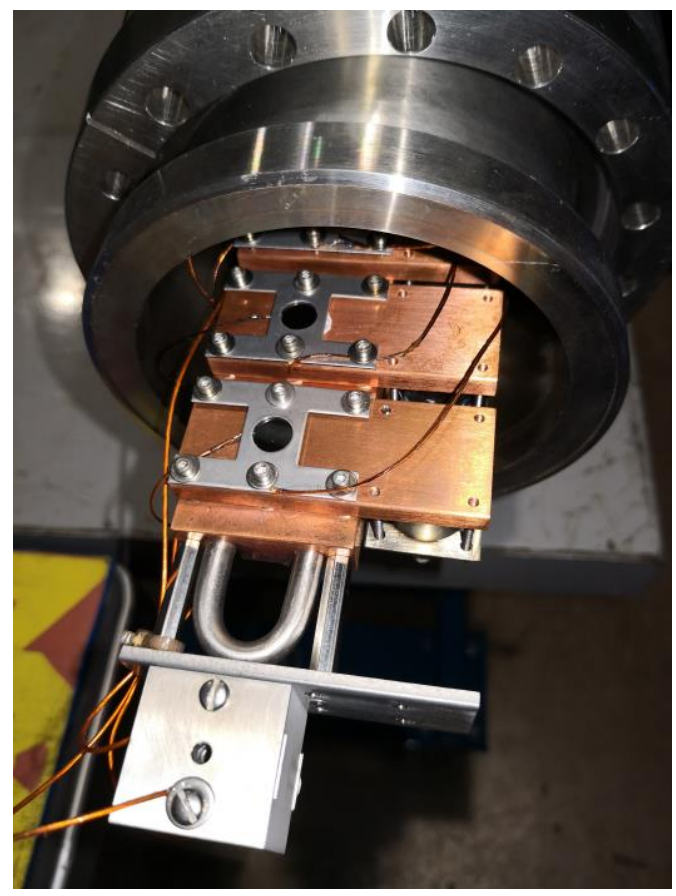

\begin{tabular}{|c|c|c|}
\hline & Sample A & Sample B \\
\hline Initial grain size $(\mu \mathrm{m})$ & 10 & 70 \\
\hline $\begin{array}{l}\text { Irradiation } \\
\text { temperature }\left({ }^{\circ} \mathrm{C}\right)\end{array}$ & 350 & 500 \\
\hline Ion fluence (ions $/ \mathrm{cm}^{2}$ ) & $7.22 \times 10^{17}$ & $7.94 \times 10^{17}$ \\
\hline $\begin{array}{l}\text { dpa (SRIM } \\
\text { calculations) }\end{array}$ & 1357 & 1492.7 \\
\hline$\%$ FIMA & 5.35 & 5.88 \\
\hline
\end{tabular}

Samples fabricated at RPI using SPS

Samples irradiated at ANL ATLAS beamline with 84

$\mathrm{MeV}$ Xe 


\section{High energy Xe ion-irradiation to mimic HBS formation (2/4)}

\section{Hillocks}

Restructured area with grain subdivision

Intermediate zone with damage gradient

Bubble formation

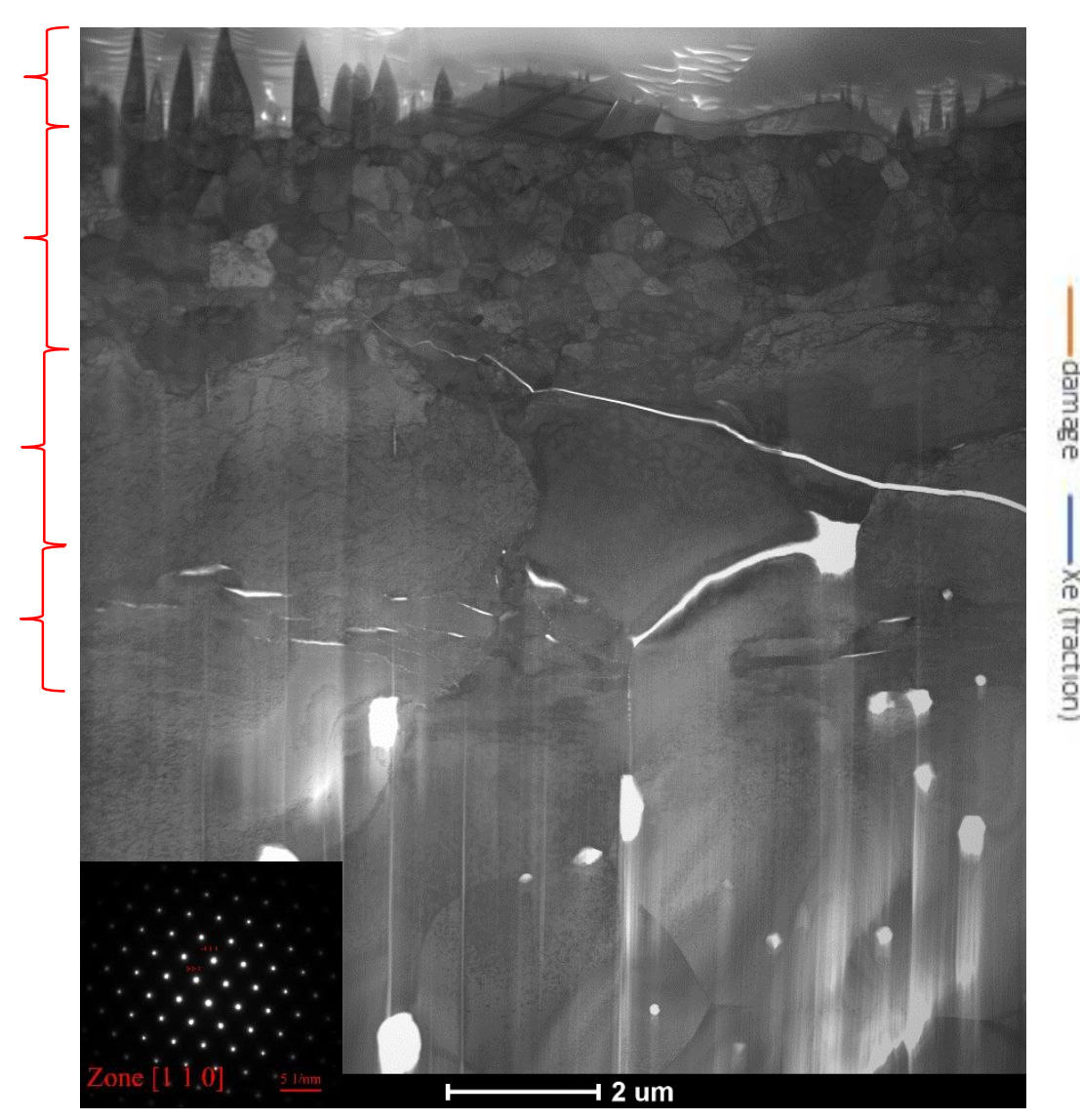

Sampled at peak beam fluence $\left(7.22 \times 10^{17}\right.$ ions $\left./ \mathrm{cm}^{2}\right)$

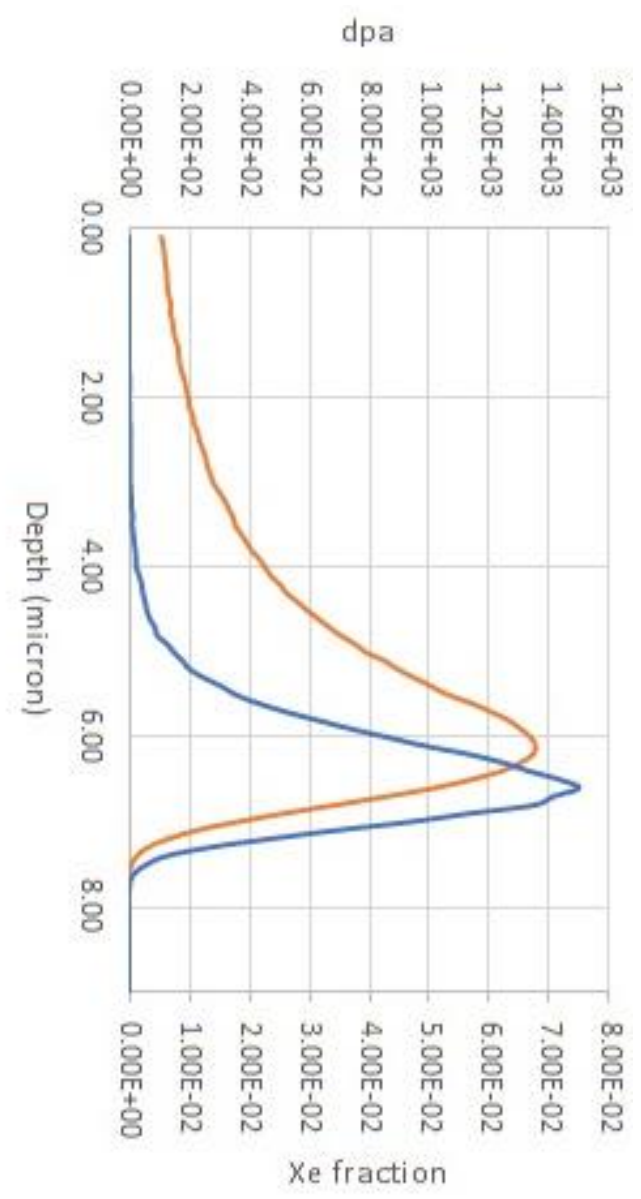




\section{High energy Xe ion-irradiation to mimic HBS} formation (3/4)

Hillocks

Grain subdivision

Intermediate zone with
damage gradient
Bubble formation
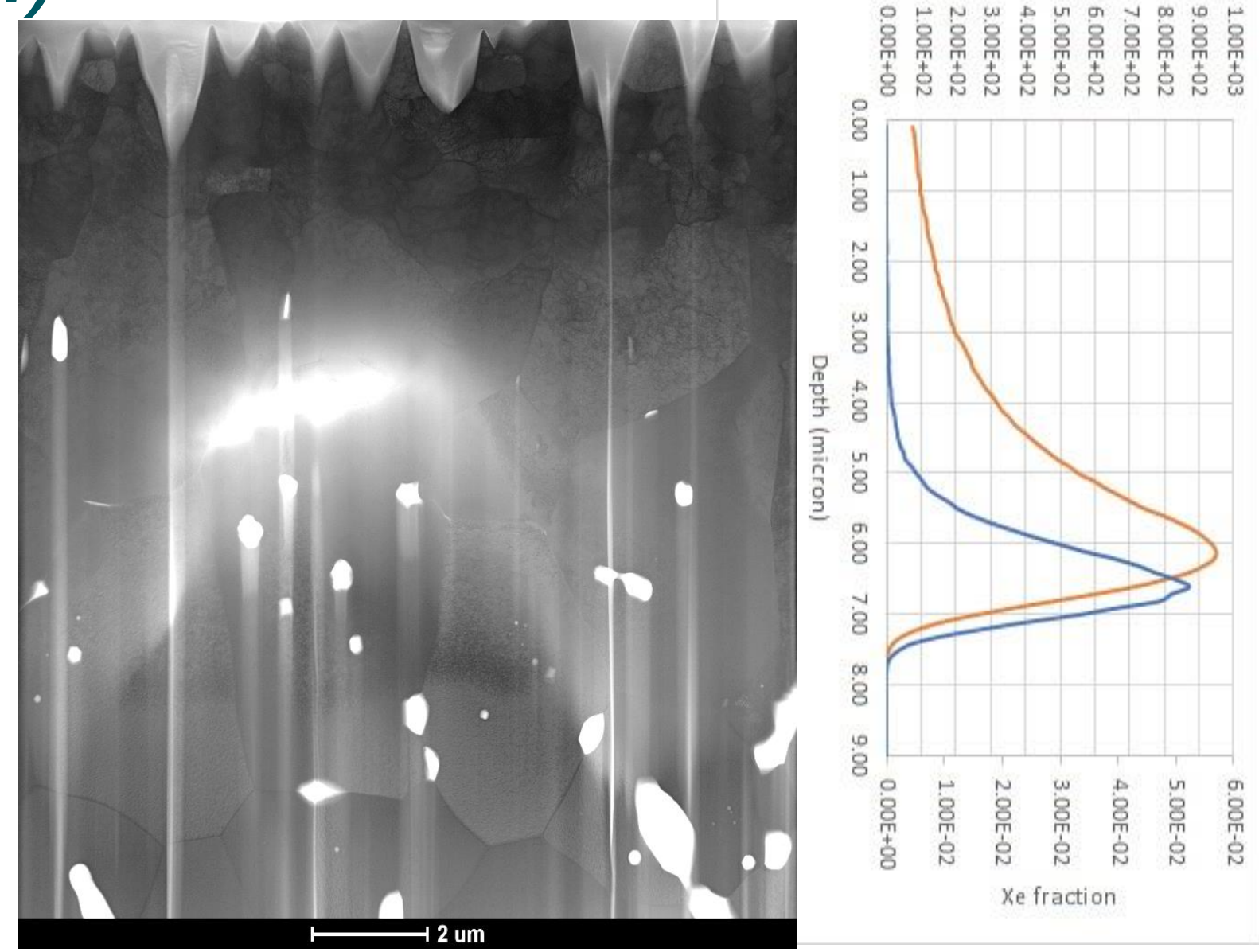

Sampled at $\approx 70 \%$ peak beam fluence $\left(5.05 \times 10^{17}\right.$ ions $/ \mathrm{cm}^{2}$ ) 


\section{High energy Xe ion-irradiation to mimic HBS formation (4/4)}
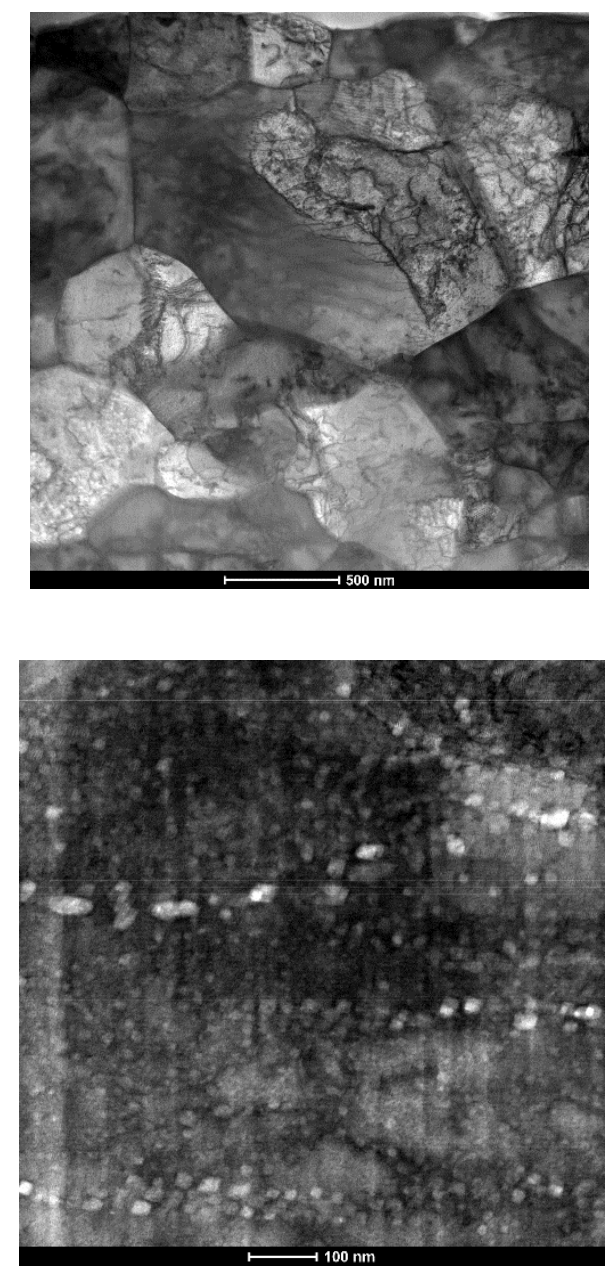

- Grain polygonization in $\mathrm{UO}_{2}$ by swift heavy ion irradiation already reported by Wiss et al. in NIMB (2000)

- At the Xe peak deposition, 2 classes of bubble size observed. Results consistent with previous work by Miao et al. in Scripta Materialia (2018)

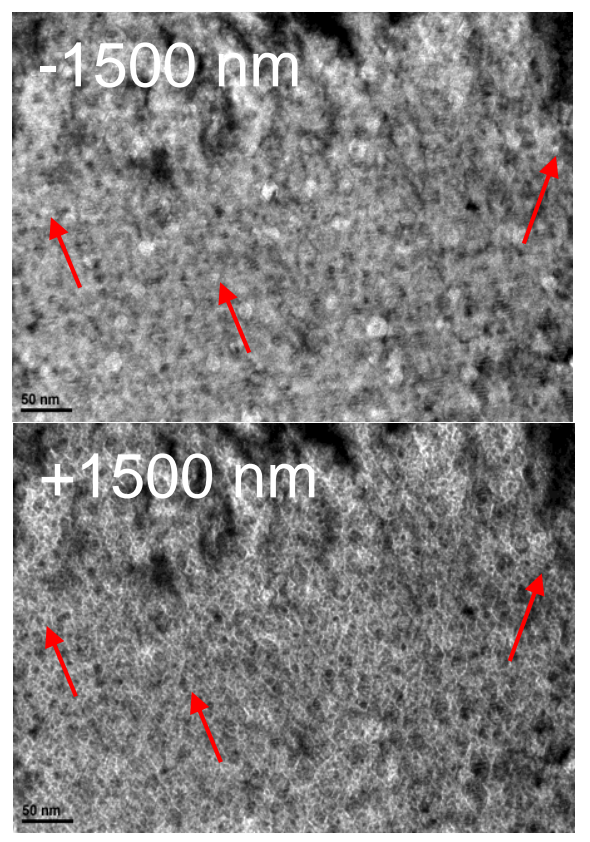

- Grain subdivision was not observed at the peak dose area, rather it occurs independently from the formation of bubbles

- Restructuring occurs at the sample surface, where electronic energy losses dominate

- Real effect or influence of sample oxidation? More characterization planned 


\section{Summary}

- Modern characterization techniques have proven to be crucial for understanding fuel microstructure evolution upon neutron damage accumulation

- EBSD analyses reveal restructuring "in-fieri" at intermediate radial locations

- Commercial fuel characterization is used to evaluate microstructural changes, but deconvolution of effects is difficult

- Separate-effect testing using ion-implantation is a unique tool to study singleparameter effects

- Grain subdivision observed independently of bubble formation. Role of electronic energy losses in $\mathrm{UO}_{2}$ grain subdivision? 


\section{Acknowledgements}

This work was funded by the US Department of Energy - Nuclear Energy Office (DOE-NE) Nuclear Technology Research and Development (NTRD), Advanced Fuels Campaign (AFC) and Laboratory Directed Research and Development (LDRD) Program (Project 19A39-061)

We would like to acknowledge Framatome for giving permission to use the irradiated commercial specimen

We also acknowledge J. Merrill, J. Wilding, K. Williams, B. Frickey, J. Stanek, A. Pomo, P. Bragg, M. Taylor, E. Perez, N. Bolender (INL) for technical and administrative support during various stages of these project

Contacts: 
